\title{
Icosapent Ethyl Effects on Fatty Acid Profiles in Statin- Treated Patients With High Triglycerides: The Randomized, Placebo-controlled ANCHOR Study
}

\author{
Christie M. Ballantyne - Mehar S. Manku • Harold E. Bays • \\ Sephy Philip · Craig Granowitz · Ralph T. Doyle Jr • Rebecca A. Juliano
}

Received: December 20, 2018 / Published online: February 20, 2019

(C) The Author(s) 2019

\begin{abstract}
Introduction: Fatty acid content in plasma and red blood cells (RBCs) may provide insight into potential physiologic benefits of omega-3 fatty acids. Icosapent ethyl is a pure prescription form of eicosapentaenoic acid (EPA) ethyl ester approved by the US Food and Drug Administration at a dose of $4 \mathrm{~g} /$ day as an adjunct to diet to reduce triglyceride levels in adults with severe $(\geq 500 \mathrm{mg} / \mathrm{dl})$ hypertriglyceridemia.
\end{abstract}

Methods: This was a prespecified exploratory subset analysis of the ANCHOR study, which

Enhanced digital features To view enhanced digital features for this article go to https://doi.org/10.6084/ m9.figshare.7643018.

C. M. Ballantyne $(\bowtie)$

Department of Medicine, Baylor College of

Medicine and the Houston Methodist DeBakey

Heart and Vascular Center, Houston, TX, USA

e-mail: cmb@bcm.tmc.edu

M. S. Manku

Amarin Pharma Inc, Bedminster, NJ, USA

H. E. Bays

Clinical Research, Louisville Metabolic and

Atherosclerosis Research Center, Louisville, KY, USA

S. Philip · C. Granowitz

Medical Affairs, Amarin Pharma Inc, Bedminster, NJ, USA

R. T. Doyle Jr · R. A. Juliano

Research and Development, Amarin Pharma Inc, Bedminster, NJ, USA randomized 702 statin-treated patients at increased cardiovascular risk with triglycerides 200-499 mg/dl and controlled low-density lipoprotein cholesterol (40-99 mg/dl). This analysis examined effects of icosapent ethyl $4 \mathrm{~g} /$ day versus placebo on fatty acid levels in plasma and RBCs using a gas chromatograph assay method with flame ionization detector.

Results: In plasma, treatment with icosapent ethyl $4 \mathrm{~g}$ /day resulted in significant increases versus placebo in the mean concentrations of EPA $(+635 \% ; P<0.0001)$ and its metabolite, docosapentaenoic acid n-3 (+ 143\%; $P<0.0001)$ with no significant change in docosahexaenoic acid. Treatment with icosapent ethyl $4 \mathrm{~g} /$ day versus placebo also resulted in significant decreases in the omega- 6 fatty acids linoleic acid $(-25 \%)$ and arachidonic acid (AA; - 31\%), as well as the AA/EPA ratio (-91\%). Icosapent ethyl $4 \mathrm{~g}$ /day also decreased the omega-9 fatty acid oleic acid (-29\%) and the saturated fatty acids palmitic acid (-23\%) and stearic acid $(-16 \%)$ (all $P<0.0001)$. Results were similar for RBCs.

Conclusions: Icosapent ethyl $4 \mathrm{~g}$ /day significantly increased EPA and produced other potentially beneficial shifts in fatty acids in plasma and RBCs versus placebo.

Trial Registration: ClinicalTrials.gov Identifier, NCT01047501

Funding: Amarin Pharma Inc.

Plain Language Summary: Plain language summary available for this article. 
Keywords: Arachidonic acid; Eicosapentaenoic acid; Fatty acids; Hypertriglyceridemia; Icosapent ethyl; Omega-3 fatty acids; Omega- 6 fatty acids

\section{PLAIN LANGUAGE SUMMARY}

Fatty acids are an important energy substrate, but specific fatty acids also play consequential and distinct roles in cellular function and in the development and progression of cardiovascular disease. Intake of omega-3 fatty (n-3) acids such as eicosapentaenoic acid (EPA) and docosahexaenoic acid (DHA) may differentially affect cardiovascular risk. Therefore, understanding the effects of specific fatty acid therapies on fatty acid profiles in plasma and red blood cells (RBCs) may provide insight into how given therapies may affect cardiovascular disease. We examined the effects of icosapent ethyl $4 \mathrm{~g} /$ day, a high-purity prescription form of EPA, versus a placebo on fatty acid levels in blood. Patients were treated with statins to control low-density lipoprotein cholesterol, but still had high triglycerides. Gas chromatography was used to measure omega-3, omega-6, saturated, and other fatty acid levels in plasma and RBCs. In plasma, icosapent ethyl $4 \mathrm{~g}$ /day increased EPA concentrations $635 \%$ and EPA's omega-3 metabolite docosapentaenoic acid (DPA) 143\%, and decreased levels of the omega- 6 fatty acid arachidonic acid (AA) $31 \%$, the AA to EPA ratio $91 \%$, and saturated fatty acid levels (stearic and palmitic acids) 16 and 23\%, respectively (all comparisons statistically significant versus placebo), while DHA levels did not change. Trends were similar for RBCs, except DHA was reduced $6 \%$ and there were no changes in stearic or palmitic acids. Results provide insight into the potential cardiovascular benefits of icosapent ethyl $4 \mathrm{~g} /$ day and how they might be related to, or distinct from, changes in other fatty acid levels.

\section{INTRODUCTION}

Fatty acid classes include saturated, monounsaturated, and polyunsaturated, such as omega-
9, omega- 6 , and omega-3. Fatty acids are not only sources of energy but are also important physiologic modulators. The proportions and concentrations of various fatty acids in plasma and red blood cell (RBC) membranes are associated with cardiovascular risk and the production of inflammatory mediators [1-7], and substantial intake of omega- 3 fatty acids may affect the fatty acid composition of plasma and RBC membranes $[1,4]$. Studies of intervention with the omega-3 fatty acids eicosapentaenoic acid (EPA) and docosahexaenoic acid (DHA) suggest a variety of potential cardiovascular-related physiological benefits: they lower plasma triglyceride (TG) levels, improve other atherogenic lipid levels, and may have beneficial effects on inflammation, vascular function, resting heart rate, blood pressure, myocardial filling and efficiency, arrhythmia, plaque stabilization, and platelet aggregation [7-9]. Despite some similarities, there are also differences in EPA and DHA, such as an observed increase in low-density lipoprotein cholesterol (Ldl-C) in some patients administered DHA-containing omega-3 mixtures that is not observed with EPA-only therapy [10-12]. Analysis of fatty acid levels in plasma and RBCs may provide greater understanding of the potential physiologic effects of omega-3 fatty acid treatment on lipid levels [13] and effects beyond lipids.

Icosapent ethyl (Vascepa; Amarin Pharma Inc, Bedminster, NJ, USA) is a high-purity prescription form of EPA ethyl ester approved by the US Food and Drug Administration at a dose of $4 \mathrm{~g}$ /day as an adjunct to diet to reduce TG levels in adults with severe $(\geq 500 \mathrm{mg} / \mathrm{dl})$ hypertriglyceridemia [14]. Compared with placebo in the 12-week, randomized, double-blind, placebo-controlled MARINE study (very high TG levels of $500-2000 \mathrm{mg} / \mathrm{dl}$ ) and ANCHOR study (TG levels 200-499 mg/dl despite statin stabilization), icosapent ethyl significantly improved levels of TGs and other atherogenic lipid, lipoprotein, and inflammatory parameters without raising the levels of Ldl-C [12, 15-19]. In these studies, icosapent ethyl was safe and generally well tolerated, with a safety profile similar to that of placebo $[12,14,17]$.

This analysis evaluated data from the ANCHOR study to determine the effects of 
icosapent ethyl at the approved dose of $4 \mathrm{~g}$ /day on fatty acid levels in plasma and RBCs in statin-treated patients with persistently high TGs.

\section{METHODS}

This prespecified exploratory analysis evaluated fatty acid levels in plasma and RBC membranes in a subset of patients from the ANCHOR study. Details of the ANCHOR study were previously published [17]. In brief, ANCHOR was a phase 3, multicenter, 12-week, double-blind, randomized, placebo-controlled study in patients at high risk for cardiovascular disease who had high TG levels while on stable statin therapy (with or without ezetimibe) to control Ldl-C levels. Patients aged $>18$ years entered a 4 - to 6-week lead-in period of diet, lifestyle, and statin stabilization during which nonstatin lipid treatments were washed out. This lead-in period was followed by a 2- to 3-week lipid-qualifying period (fasting TGs: $200-499 \mathrm{mg} / \mathrm{dl}$; fasting LdlC: $40-99 \mathrm{mg} / \mathrm{dl}$ ). Eligible patients were randomized (1:1:1) to a 12-week double-blind treatment period with either icosapent ethyl $4 \mathrm{~g} /$ day, $2 \mathrm{~g} /$ day, or placebo; this report focuses on the FDA-approved dose of $4 \mathrm{~g}$ /day versus placebo. The protocol was approved by the appropriate institutional review boards and patients provided informed written consent prior to enrollment. All procedures performed in this study were in accordance with duly constituted Institutional Review Boards for each study center and with the 1964 Declaration of Helsinki and its later amendments. Informed consent was obtained from all individual participants included in the study.

As prespecified in the study protocol as exploratory efficacy parameters, the method used to measure and analyze fatty acid levels in plasma and RBCs from patients in the ANCHOR study is similar to that previously published [13]. The method involved a validated gas chromatograph assay method with flame ionization detector (GC/FID; performed by Mylnefield Research Services Ltd, Scottish Crop Research Institute, Dundee, Scotland, UK). Briefly, fasting blood samples were collected prior to the administration of study drug at baseline and week 12. After centrifugation of the fasting blood samples, the plasma layer was removed for analysis. The buffy coat was removed from the remaining fraction and the RBCs were then washed with an equal volume of $0.9 \%$ saline or phosphate buffered saline. Following centrifugation, the saline wash was removed and the remaining RBC samples and the previously removed plasma samples were stored frozen at $-80^{\circ} \mathrm{C}$. Lipids were extracted from plasma and RBC suspensions and converted into fatty acid methyl esters for GC/FID analysis and determination of the fatty acid profile. The GC/FID assay measures total fatty acid concentrations including unesterified fatty acids and esterified fatty acids that are incorporated in circulating phospholipids, triacylglycerols, and cholesteryl esters. The lipid fraction was isolated by centrifugation after adding acid/methanol/chloroform. Lipid hydrolysis and transmethylation were accomplished with acid/methanol overnight at $50^{\circ} \mathrm{C}$, followed by extraction with chloroform/ methanol and an acidified salt wash to remove non-lipid contaminants. Fatty acids were quantified with FID and the lower limit of quantitation was $3 \mu \mathrm{g} / \mathrm{g}$ in plasma and RBCs. Concentrations were expressed as micrograms of each fatty acid per gram of sample $(\mu \mathrm{g} / \mathrm{g})$ or as a proportion in mole percent $(\mathrm{mol} \%)$, which is the molar concentration of each fatty acid expressed as a percentage of the total molar fatty acid concentration of four classes (saturated, monounsaturated, omega-3, and omega6 fatty acids). Per study protocol, the prespecified exploratory fatty acid analysis was conducted in the first 72 patients with available complete sample sets. Samples from the other patients in the study were banked for later analysis per protocol. Of the banked samples, 85 were assayed subsequently, of which 83 had complete sample sets for a total of 155 patients included in the analysis (4 g/day, $n=50$; $2 \mathrm{~g} /$ day, $n=52$; placebo, $n=53$ ). Outliers were identified and excluded for each fatty acid parameter for each treatment group. Outliers were defined as percent change values $<\mathrm{Q} 1-1.5 \cdot \mathrm{IQR}$ or $>\mathrm{Q} 3+1.5 \cdot \mathrm{IQR}$, where Q1 and Q3 are first and third quartiles of the percent change values for the treatment 
group and IQR is the interquartile range (Q3-Q1). Because outliers were defined separately for each fatty acid measure, the numbers of subjects included for some parameters were different.

Statistical analyses were performed as previously defined [13]. An analysis of covariance (ANCOVA) model with treatment, gender, type of statin therapy, and presence of diabetes at randomization as factors and the associated baseline value as a covariate for each fatty acid parameter was used for comparisons between icosapent ethyl and placebo groups. Summary statistics [ $n$, mean, and standard deviation (SD)] were provided. For each treatment group and each comparison between icosapent ethyl and placebo groups, the least squares (LS) means and standard errors (SEs) were calculated. Linear contrasts of the ANCOVA model were used to obtain the LS estimates and $P$ values for the comparisons of icosapent ethyl with placebo, with a significance level of $P=0.05$. The statistical analyses for all fatty acids were considered as exploratory endpoints in the formal study statistical analysis plan.

\section{RESULTS}

The ANCHOR trial randomized 702 statin-treated patients to icosapent ethyl $4 \mathrm{~g} /$ day, $2 \mathrm{~g} /$ day, or placebo. Reported here are fatty acid findings in patients receiving icosapent ethyl $4 \mathrm{~g}$ /day $(n=50)$ or placebo $(n=52)$ in the ANCHOR study with available fatty acid data. Baseline characteristics were similar between the two treatment groups in the randomized population [17].

Key omega-3 fatty acids of interest included EPA (C20:5n-3), DHA (C22:6n-3), and docosapentaenoic acid (DPA, C22:5n-3; an EPA metabolite and precursor to DHA), the omega-6 fatty acid, arachidonic acid (AA, C20:4n-6), and the proportion of total omega-3, omega- 6 , monounsaturated, and saturated fatty acids to the total fatty acid pool measured.

Respective mean molar EPA plasma concentrations $(\mathrm{mol} \% \pm \mathrm{SD})$ in the icosapent ethyl and placebo groups were $0.4 \pm 0.1 \%$ and $0.5 \pm 0.6 \%$ at baseline and $3.6 \pm 1.4 \%$ and
$0.5 \pm 0.5 \%$ at week 12 , respectively. Icosapent ethyl $4 \mathrm{~g}$ /day produced significant mean percent increases in the plasma and RBC concentrations of EPA and its DPA metabolite and significant decreases in AA concentrations and the AA/EPA ratio (all $P<0.0001$; Tables 1,2 ) versus placebo. DHA concentrations did not change significantly in plasma, but showed a small significant $(P<0.02)$ mean percent decrease with icosapent ethyl $4 \mathrm{~g}$ /day versus placebo in RBCs (Tables 1 and 2).

Compared with placebo, icosapent ethyl $4 \mathrm{~g}$ /day resulted in substantial and statistically significant mean percent increases in total omega-3 fatty acid concentrations in plasma (142.22\%) and RBCs (89.38\%; all $P<0.0001$; Table 3), and smaller, but significant decreases in total omega- 6 fatty acid and monounsaturated fatty acid concentrations (all $P<0.05$ ). A slight increase in saturated fatty acids was observed in RBCs $(3.13 \% ; P=0.0011)$ in the icosapent ethyl $4 \mathrm{~g}$ /day group versus placebo, with no significant change in plasma (Table 3 ).

These fatty acids described above, together with four additional fatty acids found in Tables 1 and 2 (palmitic, C16:0; stearic, C18:0; oleic, C18:1n-9; and linoleic acids, C18:2n-6), are either the most abundant in terms of mass contribution to the total lipid concentration (and thus major sources of energy) or they are less abundant but have important physiological characteristics, even at low concentrations relative to the abundant fatty acids. In plasma, significant reductions were observed in palmitic, stearic, oleic, and linoleic acids compared with placebo (Table 1 ; all $P<0.001$ ); in RBCs, reductions were observed in all four of these fatty acids compared with placebo, but were only significant for linoleic acid (Table 2).

\section{DISCUSSION}

In this prespecified exploratory subset analysis of data from the 12-week, double-blind ANCHOR study, icosapent ethyl $4 \mathrm{~g}$ /day, a highpurity prescription form of the ethyl ester of EPA, significantly increased concentrations of EPA and DPA (a metabolite of EPA) in plasma and RBCs compared with placebo in patients 
Table 1 Change from baseline to week 12 in plasma concentrations of key fatty acids

\begin{tabular}{|c|c|c|c|c|c|c|c|}
\hline \multirow{2}{*}{$\begin{array}{l}\text { Plasma fatty acid } \\
\text { Concentration, } \mu \mathrm{g} / \mathrm{g}\end{array}$} & \multicolumn{3}{|c|}{ IPE $4 \mathrm{~g} /$ day } & \multicolumn{3}{|l|}{ Placebo } & \multirow{2}{*}{$\begin{array}{l}\text { Change from } \\
\text { baseline, IPE } \\
4 \mathrm{~g} / \text { day vs placebo, } \\
\%, P\end{array}$} \\
\hline & $\begin{array}{l}\text { Baseline } \\
\text { (SD) }\end{array}$ & $\begin{array}{l}\text { Week } 12 \\
\text { (SD) }\end{array}$ & $\begin{array}{l}\text { Change } \\
\text { (SE) }\end{array}$ & $\begin{array}{l}\text { Baseline } \\
\text { (SD) }\end{array}$ & $\begin{array}{l}\text { Week } 12 \\
\text { (SD) }\end{array}$ & $\begin{array}{l}\text { Change } \\
\text { (SE) }\end{array}$ & \\
\hline EPA (C20:5n-3) & 19.57 & 144.68 & 135.98 & 24.29 & 24.39 & 10.43 & 634.50 \\
\hline$n=43,51$ & $(6.410)$ & $(56.464)$ & (5.998) & $(29.025)$ & $(25.073)$ & $(5.457)$ & $<0.0001$ \\
\hline DHA (C22:6n-3) & 51.80 & 54.02 & 2.84 & 58.54 & 61.59 & 4.44 & -1.12 \\
\hline$n=48,52$ & $(15.369)$ & $(13.842)$ & $(1.852)$ & $(34.274)$ & $(32.907)$ & $(1.743)$ & 0.7820 \\
\hline DPA (C22:5n-3) & 20.19 & 50.45 & 31.79 & 21.41 & 23.83 & 3.91 & 143.27 \\
\hline$n=49,52$ & $(5.573)$ & $(14.172)$ & $(1.794)$ & $(7.999)$ & (9.403) & $(1.714)$ & $<0.0001$ \\
\hline Palmitic (C16:0) & 974.49 & 846.22 & -162.1 & 1038.7 & 1100.2 & 56.28 & -22.87 \\
\hline$n=48,51$ & $(223.707)$ & $(213.932)$ & $(44.906)$ & $(299.038)$ & $(352.507)$ & $(42.832)$ & $<0.0001$ \\
\hline Stearic (C18:0) & 278.90 & 254.07 & -30.83 & 291.88 & 304.54 & 13.52 & -16.16 \\
\hline$n=50,50$ & $(55.757)$ & $(48.217)$ & (8.149) & $(61.887)$ & (62.494) & $(7.995)$ & $<0.0001$ \\
\hline Oleic (C18:1n-9) & 979.09 & 784.41 & -220.0 & 1011.0 & 1091.5 & 66.70 & -29.48 \\
\hline$n=47,51$ & $(261.606)$ & $(208.940)$ & $(49.146)$ & $(296.705)$ & $(403.343)$ & $(46.802)$ & $<0.0001$ \\
\hline Linoleic (C18:2n-6) & 1069.4 & 922.28 & -152.5 & 1093.0 & 1221.8 & 127.42 & -24.75 \\
\hline$n=50,52$ & $(262.259)$ & $(200.324)$ & $(38.151)$ & $(258.966)$ & $(350.910)$ & $(36.433)$ & $<0.0001$ \\
\hline AA (C20:4n-6) & 282.84 & 223.26 & -60.69 & 306.96 & 328.17 & 26.25 & -30.87 \\
\hline$n=50,52$ & $(71.080)$ & $(65.530)$ & $(6.295)$ & $(74.556)$ & $(67.119)$ & $(6.124)$ & $<0.0001$ \\
\hline AA/EPA & 16.23 & 1.61 & -14.17 & 16.97 & 16.63 & 0.60 & -90.64 \\
\hline$n=47,50$ & $(6.665)$ & $(0.795)$ & $(0.556)$ & $(6.785)$ & (6.638) & $(0.532)$ & $<0.0001$ \\
\hline
\end{tabular}

${ }^{a}$ Patient numbers $(n)$ are reported for IPE $4 \mathrm{~g}$ /day and placebo, respectively

Mean and SD are reported for baseline and end-of-treatment values; least squares means and SEs are reported for changes from baseline; least squares means are reported for percent changes from baseline vs. placebo

$A A$ arachidonic acid, DHA docosahexaenoic acid, DPA docosapentaenoic acid, EPA eicosapentaenoic acid, IPE icosapent ethyl, $S D$ standard deviation, $S E$ standard error

with high cardiovascular risk and high TG concentrations while receiving statin therapy to control Ldl-C. Studies suggest that these two omega-3 fatty acids confer distinct and complementary cardiovascular benefits [20]. An increase in plasma EPA concentrations with icosapent ethyl is dose-dependent, as is its TGlowering effect [21]. Compared with placebo, icosapent ethyl significantly decreased the concentration of $\mathrm{AA}$, the $\mathrm{AA} / \mathrm{EPA}$ ratio, and total omega- 6 fatty acid concentrations and significantly increased the total omega-3 fatty acid concentrations in plasma and RBCs accordingly. As with previous investigations of EPA-only treatment, icosapent ethyl did not significantly increase DHA concentrations in plasma in the current analysis, indicating that previously reported TG lowering and changes in fatty acids were due to increases in EPA levels but not DHA levels [1, 13, 21-23]. 
Table 2 Change from baseline to week 12 in red blood cell membrane concentrations of key fatty acids

\begin{tabular}{|c|c|c|c|c|c|c|c|}
\hline \multirow{2}{*}{$\begin{array}{l}\text { RBC membrane } \\
\text { fatty acid }^{\mathrm{a}} \\
\text { Concentration, } \mu \mathrm{g} / \mathrm{g}\end{array}$} & \multicolumn{3}{|c|}{ IPE 4 g/day } & \multicolumn{3}{|l|}{ Placebo } & \multirow{2}{*}{$\begin{array}{l}\text { Change from } \\
\text { baseline, IPE } \\
4 \mathrm{~g} / \text { day vs placebo, } \\
\%, P\end{array}$} \\
\hline & $\begin{array}{l}\text { Baseline } \\
\text { (SD) }\end{array}$ & $\begin{array}{l}\text { Week } 12 \\
\text { (SD) }\end{array}$ & $\begin{array}{l}\text { Change } \\
\text { (SE) }\end{array}$ & $\begin{array}{l}\text { Baseline } \\
\text { (SD) }\end{array}$ & $\begin{array}{l}\text { Week } 12 \\
\text { (SD) }\end{array}$ & $\begin{array}{l}\text { Change } \\
\text { (SE) }\end{array}$ & \\
\hline EPA (C20:5n-3) & 6.09 & 43.28 & 39.38 & 7.09 & 6.40 & 0.84 & 618.46 \\
\hline$n=49,48$ & $(2.248)$ & $(17.957)$ & $(1.791)$ & $(3.320)$ & $(3.313)$ & $(1.730)$ & $<0.0001$ \\
\hline DHA (C22:6n-3) & 44.10 & 39.14 & -6.29 & 47.35 & 45.12 & -2.70 & -6.12 \\
\hline$n=44,47$ & (11.708) & $(8.328)$ & $(0.983)$ & $(11.696)$ & $(11.861)$ & $(0.919)$ & 0.0178 \\
\hline DPA (C22:5n-3) & 28.54 & 62.14 & 33.67 & 29.69 & 28.60 & -0.85 & 123.56 \\
\hline$n=46,46$ & $(5.757)$ & $(12.564)$ & $(1.417)$ & $(5.496)$ & $(5.146)$ & $(1.380)$ & $<0.0001$ \\
\hline Palmitic (C16:0) & 304.13 & 301.24 & -5.77 & 313.71 & 313.52 & 0.04 & -2.24 \\
\hline$n=46,50$ & $(36.930)$ & $(36.607)$ & $(4.438)$ & $(39.421)$ & $(32.643)$ & $(4.151)$ & 0.1895 \\
\hline Stearic (C18:0) & 170.95 & 170.73 & -1.15 & 173.93 & 174.73 & 0.74 & -0.71 \\
\hline$n=45,46$ & $(18.926)$ & $(18.752)$ & $(2.550)$ & $(15.024)$ & $(18.323)$ & $(2.466)$ & 0.7048 \\
\hline Oleic (C18:1n-9) & 194.52 & 188.77 & -7.74 & 198.70 & 198.42 & -1.00 & -3.15 \\
\hline$n=45,45$ & $(27.259)$ & $(23.798)$ & $(3.060)$ & $(24.650)$ & $(24.609)$ & $(2.997)$ & 0.0943 \\
\hline Linoleic $(C 18: 2 n-6)$ & 146.81 & 132.49 & -15.77 & 151.38 & 153.50 & 1.53 & -10.65 \\
\hline$n=48,48$ & $(31.853)$ & $(26.003)$ & $(3.752)$ & $(24.241)$ & $(32.521)$ & $(3.612)$ & 0.0004 \\
\hline AA $(C 20: 4 n-6)$ & 185.38 & 153.50 & -37.08 & 189.44 & 193.57 & 0.98 & -19.48 \\
\hline$n=44,47$ & $(37.044)$ & $(28.599)$ & $(3.596)$ & $(25.769)$ & $(27.707)$ & $(3.417)$ & $<0.0001$ \\
\hline AA/EPA & 31.86 & 3.61 & -26.95 & 31.44 & 33.26 & 3.10 & -98.64 \\
\hline$n=46,50$ & $(10.373)$ & $(1.700)$ & $(0.801)$ & $(11.465)$ & $(9.955)$ & $(0.767)$ & $<0.0001$ \\
\hline
\end{tabular}

${ }^{a}$ Patient numbers $(n)$ are reported for IPE $4 \mathrm{~g} /$ day and placebo, respectively

Mean and SD are reported for baseline and end-of-treatment values; least squares mean and SE are reported for changes from baseline; least squares mean is reported for percent changes from baseline vs. placebo

$A A$ arachidonic acid, $D H A$ docosahexaenoic acid, $D P A$ docosapentaenoic acid, $E P A$ eicosapentaenoic acid, IPE icosapent ethyl, $R B C$ red blood cell, $S D$ standard deviation, $S E$ standard error

Icosapent ethyl had similar effects on plasma and RBC fatty acid levels in an analogous prespecified exploratory subset analysis of the MARINE study, indicating consistency across two patient populations. In the MARINE study, icosapent ethyl $4 \mathrm{~g} /$ day increased plasma concentrations of EPA by $792 \%$, DPA by $151 \%$, and the ratio of omega-3/omega- 6 fatty acids by $146 \%$ while decreasing AA by $27 \%$ and the AA/ EPA ratio by $99 \%$ compared with placebo (all
$P<0.0001)$ [13]. In RBCs, icosapent ethyl $4 \mathrm{~g} /$ day increased EPA by $490 \%$, DPA by $106 \%$, and the omega-3/omega- 6 fatty acid ratio by $107 \%$ and decreased AA by $16 \%$ and the AA/EPA ratio by $102 \%$ (all $P<0.0001$ ) compared with placebo. No significant changes in DHA concentrations were observed in plasma or RBCs.

Similar results were found in relative changes in plasma EPA levels in ANCHOR using liquid chromatography-tandem mass spectrometry 
Table 3 Percent change in the proportion of fatty acid classes to total fatty acids

\begin{tabular}{|c|c|c|c|c|c|c|c|}
\hline \multirow{2}{*}{$\begin{array}{l}\text { Fatty acid class } \\
\text { Proportion of } \\
\text { total, } \%^{b}\end{array}$} & \multicolumn{3}{|c|}{ IPE $4 \mathrm{~g} / \mathrm{day}$} & \multicolumn{3}{|l|}{ Placebo } & \multirow{2}{*}{$\begin{array}{l}\text { Change from } \\
\text { baseline, IPE } \\
4 \mathrm{~g} / \text { day vs placebo, } \\
\%, P\end{array}$} \\
\hline & $\begin{array}{l}\text { Baseline } \\
\text { (SD) }\end{array}$ & $\begin{array}{l}\text { Week } 12 \\
\text { (SD) }\end{array}$ & $\begin{array}{l}\text { Change } \\
\text { (SE) }\end{array}$ & $\begin{array}{l}\text { Baseline } \\
\text { (SD) }\end{array}$ & $\begin{array}{l}\text { Week } 12 \\
\text { (SD) }\end{array}$ & $\begin{array}{l}\text { Change } \\
\text { (SE) }\end{array}$ & \\
\hline \multicolumn{8}{|l|}{ Plasma } \\
\hline Saturated & 33.79 & 33.59 & -0.54 & 34.20 & 33.63 & -0.69 & 0.48 \\
\hline$n=50,53$ & $(2.543)$ & $(2.389)$ & $(0.312)$ & $(2.488)$ & $(2.357)$ & $(0.297)$ & 0.6611 \\
\hline Monounsaturated & 28.63 & 26.72 & -2.04 & 28.67 & 28.40 & -0.38 & -5.58 \\
\hline$n=50,53$ & $(2.770)$ & $(2.976)$ & $(0.442)$ & $(2.586)$ & $(3.301)$ & $(0.421)$ & 0.0038 \\
\hline Total n-3 & 2.95 & 7.16 & 4.45 & 3.11 & 3.12 & 0.25 & 142.22 \\
\hline$n=50,53$ & $(0.479)$ & $(1.978)$ & $(0.194)$ & $(1.297)$ & $(1.136)$ & $(0.185)$ & $<0.0001$ \\
\hline Total n-6 & 34.63 & 32.54 & -1.85 & 34.02 & 34.85 & 0.79 & -7.66 \\
\hline$n=50,53$ & $(3.910)$ & $(3.429)$ & $(0.520)$ & $(3.350)$ & $(3.840)$ & $(0.494)$ & $<0.0001$ \\
\hline \multicolumn{8}{|l|}{ RBC membranes } \\
\hline Saturated & 43.35 & 44.15 & 0.47 & 43.82 & 42.98 & -0.88 & 3.13 \\
\hline$n=50,53$ & $(2.165)$ & $(3.010)$ & $(0.342)$ & $(2.594)$ & $(1.724)$ & $(0.326)$ & 0.0011 \\
\hline Monounsaturated & 20.43 & 19.79 & -0.46 & 19.89 & 20.03 & 0.05 & -2.65 \\
\hline$n=50,53$ & (2.199) & $(1.688)$ & $(0.227)$ & $(1.587)$ & $(1.848)$ & $(0.215)$ & 0.0401 \\
\hline Total n-3 & 5.46 & 10.22 & 4.93 & 5.72 & 5.52 & -0.01 & 89.38 \\
\hline$n=50,53$ & $(1.130)$ & $(2.484)$ & $(0.242)$ & $(1.185)$ & $(1.116)$ & $(0.231)$ & $<0.0001$ \\
\hline Total n-6 & 30.75 & 25.84 & -4.91 & 30.58 & 31.47 & 0.81 & -19.60 \\
\hline$n=50,53$ & $(1.967)$ & $(3.165)$ & $(0.365)$ & $(3.051)$ & $(2.178)$ & $(0.348)$ & $<0.0001$ \\
\hline
\end{tabular}

${ }^{a}$ Patient numbers $(n)$ are reported for icosapent ethyl $4 \mathrm{~g} /$ day and placebo, respectively

b Proportion of total where total $=$ sum of omega-3, omega- 6 , monounsaturated, and saturated fatty acids

Mean and SD are reported for baseline and end-of-treatment values; least squares mean and SE are reported for changes from baseline; least squares mean is reported for percent changes from baseline vs. placebo

Saturated fatty acids: sum of myristic, palmitic, stearic, arachidic, behenic, and lignoceric acids

Monounsaturated fatty acids: myristoleic, palmitoleic, $c i s$-vaccenic, oleic, gondoic/gadoleic, erucic, and nervonic acids n-6 fatty acids: sum of linoleic, $\gamma$-linolenic, eicosadienoic, dihomo- $\gamma$-linolenic, $n-6$ arachidonic, adrenic, and n- 6 docosapentaenoic acids

n-3 fatty acids: sum of $\alpha$-linolenic, stearidonic, eicosatrienoic, $n-3$ arachidonic, eicosapentaenoic, $n-3$ docosapentaenoic, and docosahexaenoic acids

$I P E$ icosapent ethyl, $R B C$ red blood cell, $S D$ standard deviation, $S E$ standard error

(LC-MS/MS), another established fatty acid analysis method [21]. By LC-MS/MS, after 12 weeks of treatment with icosapent ethyl $4 \mathrm{~g} /$ day, EPA increased from a mean (SD) baseline level of $28.1(18.8) \mu \mathrm{g} / \mathrm{ml}$ to 182.6 (71.7) $\mu \mathrm{g} / \mathrm{ml}$ in plasma and from 11.6 (5.6) $\mu \mathrm{g} / \mathrm{ml}$ to $72.7(31.5) \mu \mathrm{g} / \mathrm{ml}$ in RBCs. 
Using bioanalytical methods similar to those used in the current analysis, a supplemental analysis of data from 15,534 patients from the Japan EPA Lipid Intervention Study (JELIS) also found significant increases in plasma levels of EPA and DPA, significant decreases in plasma levels of AA, and no increase in plasma levels of DHA with EPA ethyl ester $1.8 \mathrm{~g}$ /day [1]. The main finding of JELIS, conducted in Japanese patients receiving guideline-driven statin therapy $(N=18,645)$, was a $19 \%$ reduction in a composite endpoint of major coronary events with EPA treatment versus statin therapy alone [24]. The analysis of fatty acid plasma levels in the JELIS patients demonstrated that in the EPA group, a high plasma EPA concentration was significantly associated with a lower risk of a major coronary event but no significant association was found for the other plasma fatty acid levels investigated [1].

The potential cardiovascular benefits of icosapent ethyl $4 \mathrm{~g} /$ day have recently been evaluated in the Reduction of Cardiovascular Events with Icosapent Ethyl-Intervention Trial (REDUCE-IT; NCT01492361), which was focused on a multinational, high-risk, statintreated patient population similar in background risk and statin therapy to that in the ANCHOR study [25]. Patients $(N=8179)$ on stable statin therapy had Ldl-C controlled to between 41 and $100 \mathrm{mg} / \mathrm{dl}$, elevated TG between 135 and $499 \mathrm{mg} / \mathrm{dl}$, and either established cardiovascular disease (secondary prevention cohort) or diabetes and at least one other cardiovascular risk factor (primary prevention cohort) [25]. The primary composite endpoint was time to first occurrence of a major adverse cardiovascular event (MACE) that included cardiovascular death, nonfatal myocardial infarction, nonfatal stroke, coronary revascularization, or unstable angina requiring hospitalization. The key secondary endpoint was the first occurrence of three-point MACE consisting of cardiovascular death, nonfatal myocardial infarction, or non-fatal stroke. REDUCE-IT demonstrated a statistically significant relative risk reduction of $25 \%$ [hazard ratio (HR), $0.75 ; \quad 95 \%$ confidence interval (CI), 0.68-0.83; $P<0.001]$ for icosapent ethyl versus placebo in the primary composite endpoint and a relative risk reduction of $26 \%$ (HR, $0.74 ; 95 \%$ CI, $0.65-0.83 ; P<0.001)$ in the key secondary composite endpoint over a median follow-up of 4.9 years [25]. In REDUCE-IT, EPA was measured in serum using an LC-MS/MS method similar to that used in ANCHOR [21], with large increases in EPA, as observed in the ANCHOR plasma results presented herein [25]. Plasma EPA levels achieved in the JELIS trial were also substantially high, and JELIS also demonstrated a statistically significant reduction in cardiovascular events as discussed earlier.

The findings in this study are limited to an FDA-approved prescription formulation of EPA (icosapent ethyl) and cannot be extrapolated to other prescription omega-3 formulations containing different concentrations of DHA or other omega-3 fatty acids, or to dietary supplements, which are unregulated and contain much lower concentrations of EPA, DHA, or other fatty acids [26]. DHA may increase Ldl-C levels in some patients [27], and outcomes studies of low-dose omega-3 fatty acid dietary supplements have not found any cardiovascular benefit [28].

Icosapent ethyl is not currently FDA approved to reduce the risk of MACE, but further assessment of REDUCE-IT data will help to provide a greater understanding of the REDUCE-IT results, their place in clinical treatment, and the potential implications for fatty acid profiles and cardiovascular outcomes following icosapent ethyl treatment.

Physiologically, potential beneficial effects of omega-3 fatty acids include reducing inflammatory processes, such as those associated with cardiovascular disease, whereas omega- 6 fatty acids are associated with proinflammatory effects $[6,7,29,30]$. EPA competitively inhibits AA as a substrate in the production of eicosanoids and produces molecules that exert antiinflammatory and/or antithrombotic effects $[6,7,29]$. The EPA/AA ratio is correlated to the progression of arteriosclerotic disease and cardiovascular outcomes $[1,7,31,32]$. In the current analysis, icosapent ethyl significantly increased total omega-3 fatty acid concentrations and reduced the content of AA in both plasma and RBCs, significantly decreasing the $\mathrm{AA} / \mathrm{EPA}$ ratio. 
These potentially beneficial anti-inflammatory effects of icosapent ethyl versus placebo on plasma and RBC fatty acids are consistent with the previously reported reductions in markers of inflammation and oxidation in patients in the ANCHOR study, including high sensitivity C-reactive protein, lipoprotein-associated phospholipase $\mathrm{A}_{2}$, and oxidized LDL [16].

Limitations of the current analysis of plasma fatty acid levels include small sample size relative to the entire ANCHOR population, within-subject variability [33], and that changes in plasma lipid levels affect plasma fatty acid levels; thus, lipidlowering agents such as icosapent ethyl are expected to affect fatty acid levels. The analysis of RBC fatty acid levels in this study, however, offers additional understanding of the effects of icosapent ethyl, as RBCs are more reflective of the levels of EPA and other fatty acids in tissues compared with levels in plasma, and the concentrations of both EPA and DHA are known to be less variable in RBCs than in plasma [33]. Furthermore, the levels of EPA, DPA, and DHA in RBCs have been linked with cardiovascular risk, and thus analyses of RBC fatty acid levels offer additional insight into potential beneficial effects of omega-3 therapies. RBC levels of EPA and DPA help predict risk of 2-year mortality in myocardial infarction patients, and in an observational study, dietary intake and RBC levels of EPA and DHA were inversely related to the risk of primary cardiac arrest [2, 34]. However, it should be noted that in the current analysis, RBC fatty acid levels may have been limited by the 12-week study duration. While EPA concentration in RBC membranes may double as early as 3 days after supplementation, the lifespan of RBCs is approximately 120 days in adult humans [35]. Finally, as current guidelines favor as-high-as-tolerable statin intensity, it may be worth noting that in the overall ANCHOR population, approximately $90 \%$ of patients were on moderate- to high-intensity statin regimens.

\section{CONCLUSIONS}

Icosapent ethyl $4 \mathrm{~g} /$ day significantly increased plasma and RBC EPA concentrations and caused other potentially beneficial shifts in fatty acid plasma and RBC membrane content versus placebo.

\section{ACKNOWLEDGEMENTS}

The authors thank the patients and investigators for their participation.

Funding. The ANCHOR study was sponsored and designed by Amarin Pharma Inc., Bedminster, NJ, USA and conducted, including data collection, by Medpace, Inc., Cincinnati, Ohio, USA, with funding from Amarin Pharma Inc. Amarin Pharma Inc funded the article processing charges. The authors were responsible for the analysis and interpretation of the data and the decision to publish. All authors had full access to all of the data in this study and take complete responsibility for the integrity of the data and accuracy of the data analysis.

Medical Writing and Editorial Assistance. Medical writing and editorial assistance were provided by Elizabeth Daro-Kaftan and Jim Wood of Peloton Advantage, Parsippany, NJ, and funded by Amarin Pharma Inc., Bedminster, NJ, USA. The authors thank Megan Montgomery, PhD, of Amarin Pharma Inc. for scientific review of this manuscript.

Authorship. All named authors meet the International Committee of Medical Journal Editors (ICMJE) criteria for authorship for this article, take responsibility for the integrity of the work as a whole, and have given their approval for this version to be published.

Disclosures. Christie M. Ballantyne has received research/grant support from Abbott Diagnostics, Amarin Pharma Inc., Amgen, Eli Lilly, Esperion, Ionis, Novartis, Pfizer, Regeneron, Roche Diagnostic, Sanofi-Synthelabo, NIH, ADA, and AHA (all paid to institution, not individual), and is a consultant for Abbott Diagnostics, Amarin Pharma Inc., Amgen, AstraZeneca, Boehringer Ingelheim, Eli Lilly, Esperion, Ionis, Matinas Biopharma, Merck, 
Novartis, Pfizer, Regeneron, Roche Diagnostics, and Sanofi-Synthelabo. Mehar S. Manku is Editor Emeritus of Prostaglandins, Leukotrienes and Essential Fatty Acids and Editor-in-Chief of Medical Hypotheses, and was previously employed by Amarin Pharma Inc. at the time of the study. Neither Dr. Harold Bays nor his affiliated research center \& weight management center own pharmaceutical stocks or patents. In the past 12 months, Dr. Harold E. Bays' research site has received research grants from Amarin Pharma Inc., Amgen, Alere, Allergan, AstraZeneca, Boehringer Ingelheim, Bristol Meyers Squibb, Catabasis, Dr. Reddy, Eisai, Elcelyx, Eli Lilly, Esperion, Ferrer/Chiltern, Gemphire, Gilead, GSK, Home Access, iSpecimen, Ionis, Janssen, Johnson and Johnson, Merck, Nektar, Nichi-Iko, Novartis, NovoNordisk, Omthera, Pfizer, Regeneron, Sanofi, Selecta, Takeda, and TIMI. In the past 12 months, Dr. Harold E. Bays has served as a consultant/advisor for Alnylam, Akcea, Amgen, AstraZeneca, Eisai, Eli Lilly, Esperion, Ionis, Janssen, Johnson \& Johnson, Kowa, Merck, Novartis, Prosciento, Regeneron, and Sanofi. In the past 12 months, Dr. Harold E. Bays has served as a speaker for Amarin Pharma Inc., Amgen, Eisai, Kowa, Orexigen, Regeneron, and Sanofi. Sephy Philip is an employee and stock shareholder in Amarin Corporation, the parent company of Amarin Pharma Inc. Craig Granowitz is an employee and stock shareholder in Amarin Corporation, the parent company of Amarin Pharma Inc. Ralph T. Doyle $\mathrm{Jr}$ is an employee and stock shareholder in Amarin Corporation, the parent company of Amarin Pharma Inc. Rebecca A. Juliano is an employee and stock shareholder in Amarin Corporation, the parent company of Amarin Pharma Inc.

Compliance with Ethics Guidelines. All procedures performed in this study were in accordance with duly constituted Institutional Review Boards for each study center and with the 1964 Declaration of Helsinki and its later amendments. Informed consent was obtained from all individual participants included in the study.
Data Availability. The analytic methods included in this article may be used by other researchers for reproducing the results or replicating the procedure herein. Data and study materials are not publicly available due to proprietary elements owned by Amarin Pharma, Inc., and, therefore, cannot be broadly disclosed or made available.

Open Access. This article is distributed under the terms of the Creative Commons Attribution-NonCommercial 4.0 International License (http://creativecommons.org/licenses/ by-nc/4.0/), which permits any noncommercial use, distribution, and reproduction in any medium, provided you give appropriate credit to the original author(s) and the source, provide a link to the Creative Commons license, and indicate if changes were made.

\section{REFERENCES}

1. Itakura $\mathrm{H}$, Yokoyama $\mathrm{M}$, Matsuzaki $\mathrm{M}$, Saito $\mathrm{Y}$, Origasa H, Ishikawa $\mathrm{Y}$, et al. Relationships between plasma fatty acid composition and coronary artery disease. J Atheroscler Thromb. 2011;18(2):99-107.

2. Harris WS, Kennedy KF, O'Keefe JH Jr, Spertus JA. Red blood cell fatty acid levels improve GRACE score prediction of 2-yr mortality in patients with myocardial infarction. Int J Cardiol. 2013;168(1):53-9.

3. von Schacky C, Harris WS. Cardiovascular benefits of omega-3 fatty acids. Cardiovasc Res. 2007;73(2):310-5.

4. Harris WS, Sands SA, Windsor SL, Ali HA, Stevens TL, Magalski A, et al. Omega-3 fatty acids in cardiac biopsies from heart transplantation patients: correlation with erythrocytes and response to supplementation. Circulation. 2004;110(12):1645-9.

5. Harris WS, von Schacky C. The Omega-3 index: a new risk factor for death from coronary heart disease? Prev Med. 2004;39(1):212-20.

6. Calder PC. n-3 polyunsaturated fatty acids, inflammation, and inflammatory diseases. Am J Clin Nutr. 2006;83(6 Suppl):1505S-19S.

7. Borow KM, Nelson JR, Mason RP. Biologic plausibility, cellular effects, and molecular mechanisms 
of eicosapentaenoic acid (EPA) in atherosclerosis. Atherosclerosis. 2015;242(1):357-66.

8. Mozaffarian D, Wu JH. Omega-3 fatty acids and cardiovascular disease: effects on risk factors, molecular pathways, and clinical events. J Am Coll Cardiol. 2011;58(20):2047-67.

9. Bays H. Fish oils in the treatment of dyslipidemia and cardiovascular disease. In: Kwiterovich PO, editor. The Johns Hopkins textbook of dyslipidemia. Philadelphia, PA: Lippincott Williams \& Wolters Kluwer; 2010. p. 245-57.

10. Harris WS, Ginsberg HN, Arunakul N, Shachter NS, Windsor SL, Adams M, et al. Safety and efficacy of Omacor in severe hypertriglyceridemia. J Cardiovasc Risk. 1997;4(5-6):385-91.

11. Pownall HJ, Brauchi D, Kilinc C, Osmundsen K, Pao $\mathrm{Q}$, Payton-Ross C, et al. Correlation of serum triglyceride and its reduction by omega-3 fatty acids with lipid transfer activity and the neutral lipid compositions of high-density and low-density lipoproteins. Atherosclerosis. 1999;143(2):285-97.

12. Bays HE, Ballantyne CM, Kastelein JJ, Isaacsohn JL, Braeckman RA, Soni PN. Eicosapentaenoic acid ethyl ester (AMR101) therapy in patients with very high triglyceride levels (from the Multi-center, plAcebo-controlled, Randomized, double-blINd, 12-week study with an open-label Extension [MARINE] trial). Am J Cardiol. 2011;108(5):682-90.

13. Braeckman RA, Manku MS, Bays HE, Stirtan WG, Soni PN. Icosapent ethyl, a pure EPA omega-3 fatty acid: effects on plasma and red blood cell fatty acids in patients with very high triglyceride levels (results from the MARINE study). Prostaglandins Leukot Essent Fatty Acids. 2013;89(4):195-201.

14. Vascepa [package insert]. Bedminster, NJ: Amarin Pharma Inc.; 2017 February 2017.

15. Bays HE, Braeckman RA, Ballantyne CM, Kastelein JJ, Otvos JD, Stirtan WG, et al. Icosapent ethyl, a pure EPA omega-3 fatty acid: effects on lipoprotein particle concentration and size in patients with very high triglyceride levels (the MARINE study). J Clin Lipidol. 2012;6(6):565-72.

16. Bays HE, Ballantyne CM, Braeckman RA, Stirtan WG, Soni PN. Icosapent ethyl, a pure ethyl ester of eicosapentaenoic acid: effects on circulating markers of inflammation from the MARINE and ANCHOR studies. Am J Cardiovasc Drugs. 2013;13(1):37-46.

17. Ballantyne CM, Bays HE, Kastelein JJ, Stein E, Isaacsohn JL, Braeckman RA, et al. Efficacy and safety of eicosapentaenoic acid ethyl ester (AMR101) therapy in statin-treated patients with persistent high triglycerides (from the ANCHOR study). Am J Cardiol. 2012;110(7):984-92.

18. Ballantyne CM, Bays HE, Philip S, Doyle RTJ, Braeckman RA, Stirtan WG, et al. Icosapent ethyl (eicosapentaenoic acid ethyl ester): effects on remnant-like particle cholesterol from the MARINE and ANCHOR studies. Atherosclerosis. 2016;253:81-7.

19. Ballantyne CM, Bays HE, Braeckman RA, Philip S, Stirtan WG, Doyle RT Jr, et al. Icosapent ethyl (eicosapentaenoic acid ethyl ester): effects on plasma apolipoprotein C-III levels in patients from the MARINE and ANCHOR studies. J Clin Lipidol. $2016 ; 10(3): 635-45$.

20. Mozaffarian D, Lemaitre RN, King IB, Song X, Spiegelman D, Sacks FM, et al. Circulating longchain omega-3 fatty acids and incidence of congestive heart failure in older adults: the cardiovascular health study: a cohort study. Ann Intern Med. 2011;155(3):160-70.

21. Bays HE, Ballantyne CM, Doyle RT Jr, Juliano RA, Philip S. Icosapent ethyl: eicosapentaenoic acid concentration and triglyceride-lowering effects across clinical studies. Prostaglandins Other Lipid Mediat. 2016;125:57-64.

22. Grimsgaard S, Bonaa KH, Hansen JB, Nordoy A. Highly purified eicosapentaenoic acid and docosahexaenoic acid in humans have similar triacylglycerol-lowering effects but divergent effects on serum fatty acids. Am J Clin Nutr. 1997;66(3):649-59.

23. Nakamura N, Hamazaki T, Ohta M, Okuda K, Urakaze M, Sawazaki S, et al. Joint effects of HMG-CoA reductase inhibitors and eicosapentaenoic acids on serum lipid profile and plasma fatty acid concentrations in patients with hyperlipidemia. Int J Clin Lab Res. 1999;29(1):22-5.

24. Yokoyama M, Origasa H, Matsuzaki M, Matsuzawa Y, Saito Y, Ishikawa Y, et al. Effects of eicosapentaenoic acid on major coronary events in hypercholesterolaemic patients (JELIS): a randomised open-label, blinded endpoint analysis. Lancet. 2007;369(9567):1090-8.

25. Bhatt dl, Steg G, Miller M, Brinton EA, Jacobson TA, Ketchum SB, et al. Cardiovascular risk reduction with icosapent ethyl for hypertriglyceridemia. N Engl J Med. 2019;380(1):11-22.

26. Hilleman D, Smer A. Prescription omega-3 fatty acid products and dietary supplements are not interchangeable. Manag Care. 2016;46-52B.

27. Jacobson TA, Glickstein SB, Rowe JD, Soni PN. Effects of eicosapentaenoic acid and docosahexaenoic acid on low-density lipoprotein cholesterol 
and other lipids: a review. J Clin Lipidol. 2012;6(1):5-18.

28. Keaney JF Jr, Rosen CJ. VITAL signs for dietary supplementation to prevent cancer and heart disease. N Engl J Med. 2019;380(1):91-3.

29. James MJ, Gibson RA, Cleland LG. Dietary polyunsaturated fatty acids and inflammatory mediator production. Am J Clin Nutr. 2000;71(1 Suppl):343S-8S.

30. Simopoulos AP. Omega-6/omega-3 essential fatty acid ratio and chronic diseases. Food Rev Int. 2004;20(1):77-90.

31. Kondo T, Ogawa K, Satake T, Kitazawa M, Taki K, Sugiyama S, et al. Plasma-free eicosapentaenoic acid/arachidonic acid ratio: a possible new coronary risk factor. Clin Cardiol. 1986;9(9):413-6.
32. Ohnishi H, Saito Y. Eicosapentaenoic acid (EPA) reduces cardiovascular events: relationship with the EPA/arachidonic acid ratio. J Atheroscler Thromb. 2013;20(12):861-77.

33. Harris WS, Thomas RM. Biological variability of blood omega-3 biomarkers. Clin Biochem. 2010;43(3):338-40.

34. Siscovick DS, Raghunathan TE, King I, Weinmann S, Wicklund KG, Albright J, et al. Dietary intake and cell membrane levels of long-chain n-3 polyunsaturated fatty acids and the risk of primary cardiac arrest. JAMA. 1995;274(17):1363-7.

35. Katan MB, Deslypere JP, van Birgelen AP, Penders M, Zegwaard M. Kinetics of the incorporation of dietary fatty acids into serum cholesteryl esters, erythrocyte membranes, and adipose tissue: an 18-month controlled study. J Lipid Res. 1997;38(10):2012-22. 